\title{
Verification of Atellica 1500 and comparison with Iris urine analyser and urine culture
}

Ana Nikler*1, Helena Čičak1, Danijela Bejuk², Vanja Radišić Biljak , Ana-Maria Šimundić1,3

\author{
1Department of Medical Laboratory Diagnostics, University Hospital “Sveti Duh", Zagreb, Croatia \\ 2Department of Clinical Microbiology and Hospital Infections, University Hospital “Sveti Duh", Zagreb, Croatia \\ ${ }^{3}$ Faculty of Pharmacy and Biochemistry, University of Zagreb, Zagreb, Croatia \\ ${ }^{*}$ Corresponding author: ana.nikler@gmail.com
}

\begin{abstract}
Introduction: The aims of study were to assess: 1) performance specifications of Atellica 1500, 2) comparability of Atellica 1500 and Iris, 3) the accuracy of both analysers in their ability to detect bacteria.

Materials and methods: Carryover, linearity, precision, reproducibility, and limit of blank (LoB) verification were evaluated for erythrocyte and leukocyte counts. ICSH 2014 protocol was used for estimation of carryover, CLSI EP15-A3 for precision, and CLSI EP17 for LoB verification. Comparison for quantitative parameters was evaluated by Bland-Altman plot and Passing-Bablok regression. Qualitative parameters were evaluated by Weighted kappa analysis. Sixty-five urine samples were randomly selected and sent for urine culture which was used as reference method to determine the accuracy of bacteria detection by analysers.

Results: Analytical specifications of Atellica 1500 were successfully verified. Total of 393 samples were used for qualitative comparison, while 269 for sediment urinalysis. Bland-Altman analysis showed statistically significant proportional bias for erythrocytes and leukocytes. Passing-Bablok analysis for leukocytes pointed to significant constant and minor proportional difference, while it was not performed for erythrocytes due to significant data deviation from linearity. Kappa analysis resulted in the strongest agreements for $\mathrm{pH}$, ketones, glucose concentrations and leukocytes, while the poorest agreement for bacteria. The sensitivity and specificity of bacteria detection were: 91 (59-100)\% and 76 (66-87)\% for Atellica 1500 and $46(17-77) \%$ and 96 (87-100)\% for lris.
\end{abstract}

Conclusion: There are large differences between Atellica 1500 and lris analysers, due to which they are not comparable and can not be used interchangeably. While there was no difference in specificity of bacteria detection, Iris analyser had greater sensitivity.

Keywords: Atellica 1500 analyser; Iris analyser; urine; verification

\section{Introduction}

The urine analysis is one of the most commonly performed tests in medical laboratories. It is an important tool in diagnostic pathway for various urinary tract disorders (1). Various guidelines for the assessment of chronic kidney disease, urinary tract infections and tumor formations emphasize the importance of urinalysis as the first step in diagnostic pathway and further evaluation of patient condition (2-4).
Automation of urine analysis has been a major step forward in urine analysis, by standardizing the analysis and substantially reducing the analysis turnaround time $(5,6)$. Over the past years, automated urine analysis has become an inevitable part of routine medical laboratory practice. Automated urine analysers employ different method principles. Atellica 1500 (Siemens healthineers, Erlangen, Germany) is one of the most recent auto- 
mated urine analysers, which uses a built-in centrifuge and a camera to capture sediment images that are evaluated by a sophisticated software for classification of urine particles. The performance of Iris (Beckman Coulter, Brea, USA) urine analyser differs from Atellica 1500. Iris method is based on laminar flow digital imaging technology without previous sample centrifugation. Currently there are no studies about verification of new Atellica 1500 specifications and its comparison to Iris analyser. According to their methods differences it is questionable whether the urine results of these two analysers are even comparable. A further challenge is their accuracy and agreement with the urine culture in capability of bacteria detection.

Therefore, the aims of our study were to assess: 1) performance specifications of Atellica 1500 urine analyser: carryover, linearity, precision, reproducibility and verification of limit of blank (LoB) for erythrocyte and leukocyte counts, 2) comparability of Atellica 1500 and Iris urine analyser, and 3) the accuracy of both analysers in their ability to detect bacteria.

\section{Material and methods}

The study was performed at Department of Medical Laboratory Diagnostics of University hospital "Sveti Duh" (Zagreb, Croatia) during July 2018.

\section{Methods comparison}

Leftover urine samples consecutively collected from emergency and routine hospital departments during daily routine were used to estimate comparison of two urine analysers: Atellica 1500 (Siemens healthineers, Erlangen, Germany) and Iris (Beckman Coulter, Brea, USA). Samples were analysed within 1 hour after their collection. Atellica 1500 analyser is consisted of Clinitek Novus (urine strip analysis) and Atellica UAS 800 (automated urine sediment analyser).

Dipstick parameters included in this study were: $\mathrm{pH}$, ketones, glucose, leukocytes, nitrites, bilirubin, blood, specific gravity, proteins and urobilinogen, while yeasts, mucus, hyaline casts, epithelial cells, bacteria, erythrocyte and leukocyte count were included in urine sediment analysis.

Comparison for quantitative parameters (erythrocyte and leukocyte counts) was evaluated by Bland-Altman plot and Passing-Bablok regression. Cusum test was used to detect the deviation of data from linearity. In cases when deviation from linearity was detected, Passing-Bablok analysis was not done. Qualitative parameters were evaluated by Weighted kappa analysis.

Weighted kappa test was used to determine levels of agreement for urine test strips analysis and following urine sediment parameters: yeast, mucus, hyaline casts, epithelial cells and bacteria. The agreement was expressed as Cohen kappa value (K). Kappa value $\geq 0.60$ was considered acceptable (7). Default manufacturer's reporting categories were used. For the purpose of the agreement assessment, some categories were merged to gain at least 10 samples per category.

Urine test strips parameters which were analysed as negative and positive and their respective cutoffs are as follows: ketones ( $2 \mathrm{mmol} / \mathrm{L})$, nitrites $(16.2 \mu \mathrm{mol} / \mathrm{L})$, glucose $(14 \mathrm{mmol} / \mathrm{L})$, bilirubin $(8.5$ $\mu \mathrm{mol})$, urobilinogen $(33 \mu \mathrm{mol} / \mathrm{L})$ and proteins $(0.5$ $\mathrm{g} / \mathrm{L})$. Urine $\mathrm{pH}$ was categorized into three categories, as follows: 5.0-5.5, 6.0-6.5, 7.0-7.5 and 8.0-9.0 units, while specific gravity categories were: 1.000 $1.009,1.010-1.019$ and $>1.020$. Blood and leukocytes were divided in three categories: $\leq 33, \leq 163$, $\leq 326 \mathrm{Erc} / \mu \mathrm{L}$ and $\leq 25, \leq 75, \leq 500 \mathrm{Lkc} / \mu \mathrm{L}$. Urine sediment parameters (modified due to merging, as described above) are shown in Table 1.

Data were analysed using MedCalc 12.6.2.0 (Ostend, Belgium) statistical software and Microsoft Office Excel 2010 (Microsoft, Washington, USA).

\section{Accuracy of the ability to detect bacteria in urine}

Sixty-five urine samples that were analysed on both urine analysers were randomly selected and sent for urine culture analysis within 20 minutes after urine sediment analysis. Those urine samples were analysed in Microbiology Department of our hospital. The urine culture was used as reference 
TABLE 1. Categories of urine sediment parameters used to compare Atellica UAS 800 (Siemens healthineers, Erlangen, Germany) and Iris (Beckman Coulter, Brea, USA) urine analysers

\begin{tabular}{|c|c|c|c|}
\hline Urine sediment parameter $(p / \mu L)$ & $1^{\text {st }}$ category & $2^{\text {nd }}$ category & $3^{\text {rd }}$ category \\
\hline yeast & $\begin{array}{c}\text { Iris 0-11.0 } \\
\text { Atellica 0-4.49 }\end{array}$ & $\begin{array}{c}\text { Iris } \geq 11.1 \\
\text { Atellica } \geq 4.50\end{array}$ & $\mathrm{~N} / \mathrm{A}$ \\
\hline mucus & $\begin{array}{c}\text { Iris 0-27.5 } \\
\text { Atellica 0-396 }\end{array}$ & $\begin{array}{c}\text { Iris } \geq 27.6 \\
\text { Atellica } \geq 397\end{array}$ & $\mathrm{~N} / \mathrm{A}$ \\
\hline hyaline casts & $\begin{array}{c}\text { Iris } 0-0.7 \\
\text { Atellica 0-2.97 }\end{array}$ & $\begin{array}{c}\text { Iris } \geq 0.8 \\
\text { Atellica } \geq 2.98\end{array}$ & $\mathrm{~N} / \mathrm{A}$ \\
\hline epithelial cells & $\begin{array}{c}\text { Iris } 0-27.5 \\
\text { Atellica 0-7.52 }\end{array}$ & $\begin{array}{c}\text { Iris } 27.6-55.0 \\
\text { Atellica } 7.53-37.49\end{array}$ & $\begin{array}{c}\text { Iris } \geq 55.0 \\
\text { Atellica } \geq 37.50\end{array}$ \\
\hline bacteria & $\begin{array}{c}\text { Iris 0-6.8 } \\
\text { Atellica 0-195.03 }\end{array}$ & $\begin{array}{c}\text { Iris } 6.9-13.8 \\
\text { Atellica } 195.04-495\end{array}$ & $\begin{array}{c}\text { Iris } \geq 13.9 \\
\text { Atellica } \geq 496\end{array}$ \\
\hline
\end{tabular}

Concentration ranges of categories were defined by manufacturer. $\mathrm{p} / \mu \mathrm{L}$ - particles per microliter of urine. N/A - not available.

method to determine the accuracy of bacteria detection by Atellica UAS 800 and Iris analysers.

Each urine sample was cultured for organism quantification, identification of bacteria and susceptibility testing. Urine culture quantification was performed according to the standard practice using 10 microliter calibrated loops. After inoculating the loopful of urine to the blood agar plate on a standard way, plates were incubated overnight at $35^{\circ} \mathrm{C}$. In the urine culture, the number of colony-forming units (CFUs) per $\mathrm{mL}$ is an estimate of the number of bacteria in the sample. If uropathogens (Gram negative rods, Staphylococcus saprophyticus, Enterococcus) were isolated full identification and antimicrobial testing were done. If skin urinary microbiota was isolated (diphteroides, coagulase negative Staphylococcus spp) in pure culture and in significant number (>105 $\mathrm{CFU} / \mathrm{mL}$ ) identification and antimicrobial testing was also performed.

To determine the accuracy of bacteria detection by Atellica UAS 800 and Iris urine analysers, a bacterial count of $>10^{5} \mathrm{CFU} / \mathrm{mL}$ was considered as positive bacterial urine culture result, regardless of whether it was Gram negative or positive bacteria. The sensitivity and specificity of both analysers to detect bacteria in urine samples were calculated as showed in Table 2 . The sensitivity was calculated as "true positives" (TP) / (TP + "false negatives"
TABle 2. Contingence table of determining sensitivity and specificity of Atellica UAS 800 (Siemens healthineers, Erlangen, Germany) and Iris (Beckman Coulter, Brea, USA) urine analysers for bacteria detection compared to urine culture as reference method

\begin{tabular}{|c|c|c|c|c|}
\hline & & \multicolumn{2}{|c|}{ urine culture } & \multirow{2}{*}{$\Sigma$} \\
\hline & & positive & negative & \\
\hline \multirow{2}{*}{$\begin{array}{c}\text { urine } \\
\text { analyser }\end{array}$} & positive & TP & FP & $\mathrm{TP}+\mathrm{FP}$ \\
\hline & negative & FN & $\mathrm{TN}$ & $\mathrm{FN}+\mathrm{TN}$ \\
\hline \multicolumn{2}{|c|}{$\Sigma$} & $\mathrm{TP}+\mathrm{FN}$ & $\mathrm{FP}+\mathrm{TN}$ & Total \\
\hline
\end{tabular}

TP - true positive. FP - false positive. FN - false negative. TN true negative.

(FN)), while specificity as "true negatives" (TN) / ( $\mathrm{TN}+$ "false positives" (FP)).

Atellica UAS 800 and Iris analysers have different reporting categories for urine sediment results. Therefore, the results were provided in absolute numbers and in categories declared by both manufacturers.

\section{Carryover}

The carryover was estimated for leukocyte and erythrocyte counts in urine sediment analysis in accordance with ICSH 2014 protocol $(8,9)$. The carryover testing was performed using a sample with a high count (sample A) and another one with a 
low count (sample B) of leukocytes and erythrocytes. The sample $A$ was analysed three times (A1, $A 2, A 3)$, followed by the sample $B$ that was also analysed three times (B1, B2, B3). The carryover (\%) was calculated using the equation (B1 - B3) / (A3 $\mathrm{B} 3)$, wherein the carryover $<0.5 \%$ was considered as acceptable.

\section{Linearity, precision, reproducibility and verification of LoB}

Linearity, precision, reproducibility and verification of LoB were determined for quantitative urine sediment parameters, i.e. erythrocyte and leukocyte counts.

The linearity was assessed on two urine samples with high leukocyte and erythrocyte counts. Samples were diluted with distilled water in 1:0, 1:2, 1:4, $1: 8$ and 1:16 ratios. The observed values were plotted against the expected values wherein $R^{2}>0.99$ was considered as linear and acceptable.

Precision was determined according to CLSI EP15A3 using quality control materials (qUAntify Plus Control, BioRad, LOT 80561 and 80562, expiration date $30^{\text {th }}$ November 2019) (10). These two control materials were analysed five times per day during five consecutive days. According to manufacturer's declarations, acceptable criteria for erythrocytes was $14 \%$ and $11 \%$ for leukocytes. Two patient urine samples with high and low number of erythrocytes and leukocytes were also analysed 20 times to estimate the reproducibility on patient samples.

Limit of blank was verified according to CLSI EP17 guideline (11). Ten blank samples (distilled water) per day were analysed for 3 days. Limit of blank values for erythrocyte and leukocyte counts declared by manufacturer were $0 \mathrm{Erc} / \mu \mathrm{L}$ and $0 \mathrm{Lkc} /$ $\mu \mathrm{L}$.

\section{Determination of different sample volume effect}

To determine whether a different volume of urine sample affects the results of erythrocyte and leukocyte counts, we decided to analyse $10 \mathrm{~mL}$ as maximum volume sample as many times as Atelli- ca 1500 can analyse it without alarm for insufficient sample. Each time analyser has taken small amount of sample for analysis. Four randomly selected urine samples were used, and the recovery percentage was calculated for each sample.

\section{Results}

\section{Atellica 1500 and Iris comparison}

Total number of urine samples analysed on Iris analyser with urine test strips was 393. Urine samples without any abnormalities in urine test strips were not subjected to urine sediment analysis. Thus, sediment urinalysis was determined for 269/393 urine samples.

The results of kappa statistical analysis of agreement between Atellica 1500 (Clinitek Novus) and Iris for test strip and urine sediment analysis are presented in Table 3. The strongest agreements were observed for the following test strip parame-

TABle 3. The agreement of Atellica 1500 (Siemens healthineers, Erlangen, Germany) and Iris (Beckman Coulter, Brea, USA) automated urine analysers determined by Cohen kappa value (K) for urine test strips and sediment analysis

\begin{tabular}{|c|c|c|c|c|}
\hline & Parameter & $\begin{array}{c}\text { Kappa } \\
\text { value (к) }\end{array}$ & $\begin{array}{l}\text { Level of } \\
\text { agreement }\end{array}$ & Acceptance \\
\hline \multirow{10}{*}{ 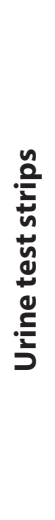 } & $\mathrm{pH}$ & 0.88 & strong & yes \\
\hline & Ketones & 0.84 & strong & yes \\
\hline & Glucose & 0.82 & strong & yes \\
\hline & Leukocytes & 0.80 & strong & yes \\
\hline & Nitrites & 0.77 & moderate & yes \\
\hline & Bilirubin & 0.72 & moderate & yes \\
\hline & Blood & 0.61 & moderate & yes \\
\hline & Specific gravity & 0.59 & weak & no \\
\hline & Proteins & 0.51 & weak & no \\
\hline & Urobilinogen & 0.08 & none & no \\
\hline \multirow{5}{*}{ 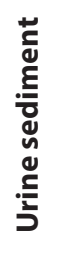 } & Yeast & 0.75 & moderate & yes \\
\hline & Mucus & 0.61 & moderate & yes \\
\hline & Hyaline casts & 0.45 & weak & no \\
\hline & Epithelial cells & 0.45 & weak & no \\
\hline & Bacteria & 0.20 & low & no \\
\hline
\end{tabular}


ters: $\mathrm{pH}$, ketones, glucose concentrations and leukocytes count. The poorest agreement was observed for bacteria in urine sediment.

Bland-Altman agreement analysis showed statistically significant proportional bias for erythrocytes and leukocytes in urine sediment (Figures 1, 2). Due to significant data deviation from linearity $(P$
$<0.010$ ), Passing-Bablok regression analysis for erythrocyte count was not performed. PassingBablok regression analysis for leukocytes pointed to the significant constant and minor proportional difference in leukocyte count in urinary sediment, between Atellica UAS 800 and Iris automated urine analyser (Figure 3).
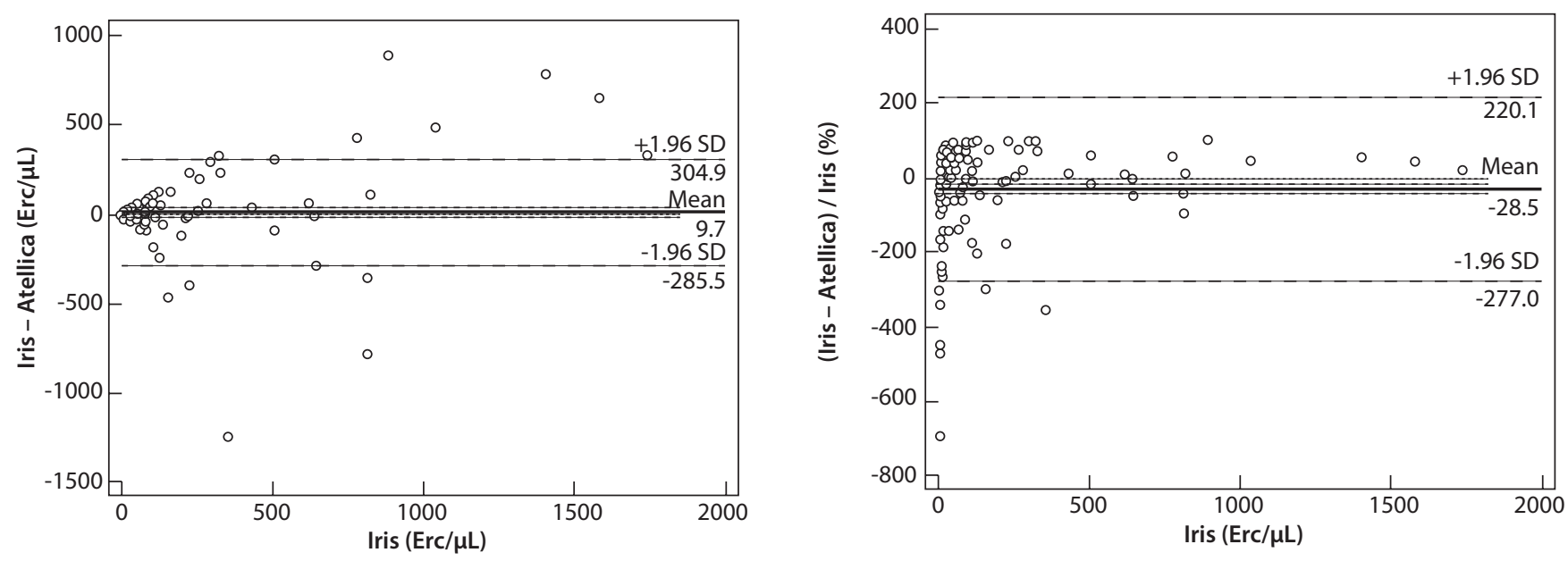

FIGURE 1. Bland-Altman plots of absolute and relative differences showing the differences in erythrocytes counts (Erc/ $\mu \mathrm{L})$ between Atellica UAS 800 (Siemens healthineers, Erlangen, Germany) and Iris (Beckman Coulter, Brea, USA) urine analysers. Solid line - mean difference. Dotted lines - 95\% confidence interval of mean difference. Dashed lines - \pm 1.96 standard deviation (SD).
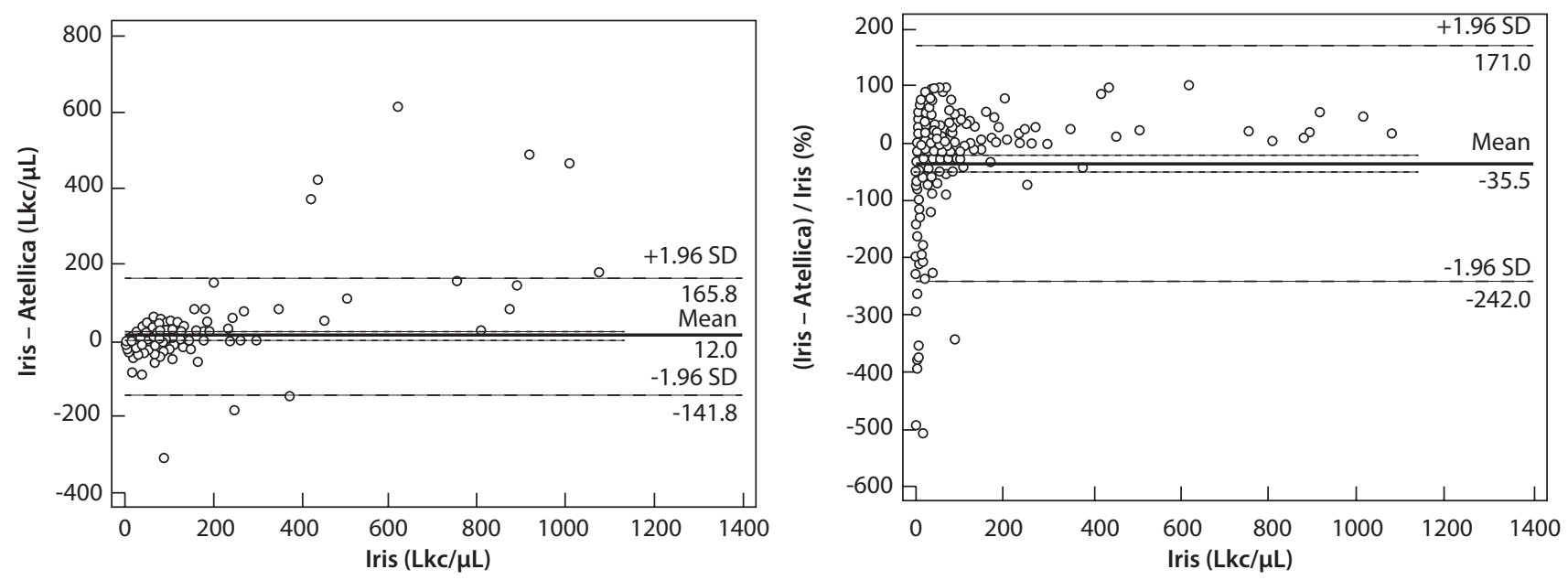

Figure 2. Bland-Altman plots of absolute and relative differences showing the differences in leukocytes counts (Lkc/ $\mu \mathrm{L}$ ) between Atellica UAS 800 (Siemens healthineers, Erlangen, Germany) and Iris (Beckman Coulter, Brea, USA) urine analysers. Solid line - mean difference. Dotted lines - 95\% confidence interval of mean difference. Dashed lines - \pm 1.96 standard deviation (SD). 


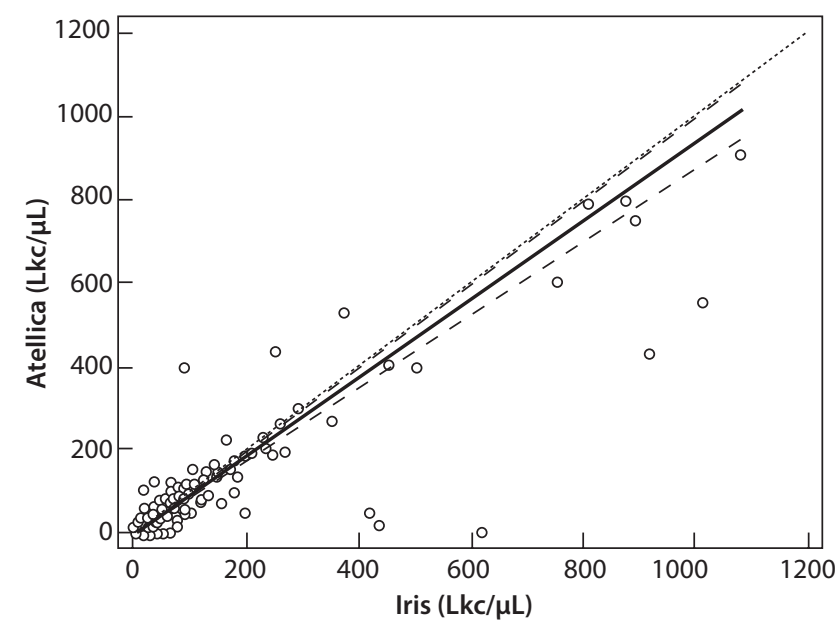

Figure 3. Passing and Bablok regression plots comparing leukocytes counts $(\mathrm{Lkc} / \mu \mathrm{L})$ on Atellica UAS 800 (Siemens healthineers, Erlangen, Germany) and Iris (Beckman Coulter, Brea, USA). Solid line - regression line. Dashed line - 95\% confidence interval of the regression line. Dotted line - identity line $(y=x)$. $y=1.38$ (0.99 to 2.13$)+0.93(0.87$ to 0.99$) x$.

\section{Accuracy of bacteria detection compared to urine culture}

The sensitivity and specificity of bacteria detection were: 91 (59-100)\% and 76 (66-87)\% for Atellica UAS 800 and 46 (17-77)\% and 96 (87-100)\% for Iris (Table 4). There was a statistically significant difference in analysers specificity, but not in the sensitivity of their bacteria detection. The results of bacteria detection comparison by Iris and Atellica 1500 urine analysers compared to urine culture results are showed in Supplementary Table 1.

\section{Other Atellica 1500 specifications}

No carryover effect was observed for erythrocytes and leukocytes counts.

Erythrocyte counts used to evaluate linearity were in the range of 62-1034 Erc/ $\mu \mathrm{L}$ along with 80-1174 $\mathrm{Lkc} / \mu \mathrm{L}$ leukocytes. Average bias for erythrocytes count was $1.87 \%$, and $0.004 \%$ for leukocytes. The coefficients of determination were within acceptance criteria of $\mathrm{R}^{2}>0.99$ : 0.9978 for erythrocytes and 0.9967 for leukocytes (Figures 4, 5).
TABle 4. Contingence table of determining sensitivity and specificity for bacteria detection of Atellica UAS 800 (Siemens healthineers, Erlangen, Germany) and Iris (Beckman Coulter, Brea, USA) urine analysers

\begin{tabular}{|c|c|c|c|c|}
\hline & & \multicolumn{2}{|c|}{ urine culture } & \multirow{2}{*}{$\Sigma$} \\
\hline & & positive & negative & \\
\hline \multirow{2}{*}{$\begin{array}{l}\text { Atellica } \\
\text { UAS } 800\end{array}$} & positive & 10 & 13 & 23 \\
\hline & negative & 1 & 41 & 42 \\
\hline \multirow[t]{3}{*}{$\Sigma$} & & 11 & 54 & 65 \\
\hline & & \multicolumn{2}{|c|}{ urine culture } & \\
\hline & & positive & negative & 2 \\
\hline \multirow{2}{*}{ Iris } & positive & 5 & 2 & 7 \\
\hline & negative & 6 & 52 & 58 \\
\hline$\Sigma$ & & 11 & 54 & 65 \\
\hline
\end{tabular}

Urine culture was used as reference method. TP - true positive. FP - false positive. FN - false negative. TN - true negative.

Method precision at $187.16 \mathrm{Erc} / \mu \mathrm{L}$ was $7.87 \%$ for erythrocytes, and $8.91 \%$ at $108.19 \mathrm{Lkc} / \mu \mathrm{L}$ for leukocytes. Coefficients of variations (CVs) for both urine parameters were within acceptance criteria. Negative control materials contained erythrocytes and leukocytes below detection limits $(<4 \mathrm{Erc} / \mu \mathrm{L}$ and $<3 \mathrm{Lkc} / \mu \mathrm{L}$ ) in each measurement and therefore no

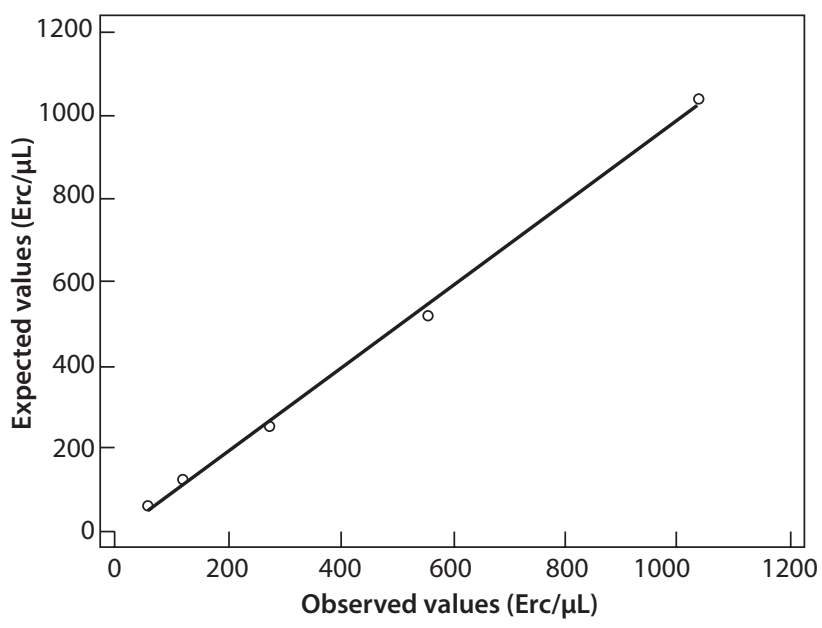

FigURE 4. Linearity plot of observed and expected erythrocyte counts (Erc/ $\mu \mathrm{L}$ ) on Atellica UAS 800 (Siemens healthineers, Erlangen, Germany) analyser. Coefficient of determination $\left(\mathrm{R}^{2}\right)$ was 0.9978 . 


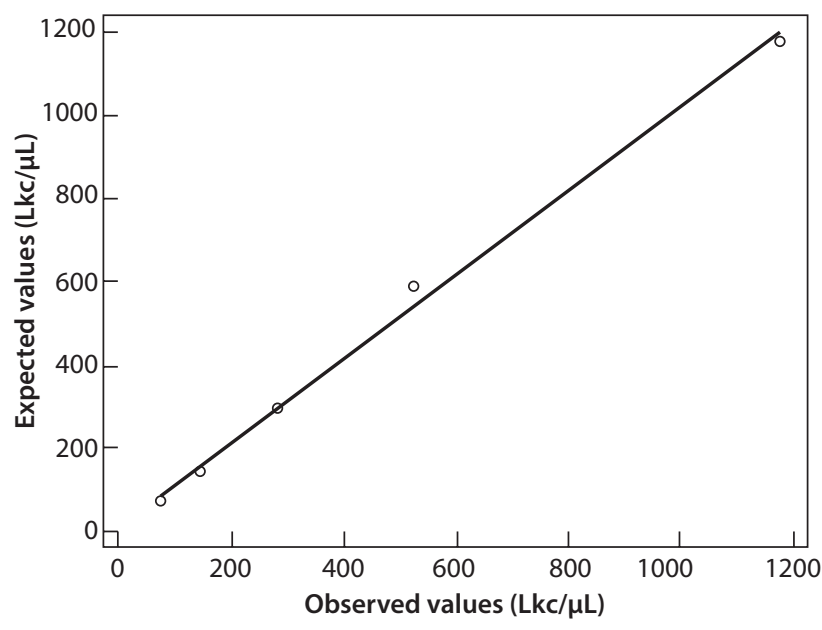

Figure 5. Linearity plot of observed and expected leukocyte

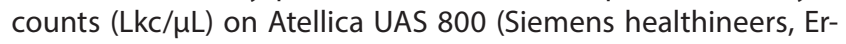
langen, Germany) analyser. Coefficient of determination $\left(R^{2}\right)$ was 0.9967.

statistical analysis can be performed. For withinrun reproducibility CVs were $7.94 \%$ for $194.44 \mathrm{Erc} /$ $\mu \mathrm{L}$ and $9.25 \%$ for $110.42 \mathrm{Lkc} / \mu \mathrm{L}$.

Limit of blank for erythrocytes and leukocytes declared by manufacturer was successfully verified. All measurements of erythrocyte and leukocyte counts were below detection limits $(<4 \mathrm{Erc} / \mu \mathrm{L}$ and $<3 \mathrm{Lkc} / \mu \mathrm{L})$.

\section{Sample volume influence on urine analysis results}

Four urine samples used to examine whether the sample volume affects the results of urine analysis were successfully analysed seven times. For each sample, the eighth analysis was not able to proceed because the analyser notified that there was not enough sample volume. Erythrocytes and leukocytes counts and recovery percentages of each sample were presented in Table 5.

\section{Discussion}

Our study has demonstrated large differences between Iris and Atellica 1500 automated urine analysers that use different methodologies. Two analysers differ in their comparability of urine test strips
TABLE 5. Recovery percentages and following erythrocytes and leukocytes counts of four urine samples used to examine the effect of sample volume on urine analysis results

\begin{tabular}{|c|c|c|c|}
\hline \multicolumn{2}{|c|}{ Erythrocytes } & \multicolumn{2}{|c|}{ Leukocytes } \\
\hline $\begin{array}{l}\text { Count } \\
\text { (Erc/ } / \mu L)\end{array}$ & $\begin{array}{c}\text { Recovery } \\
\text { (\%) }\end{array}$ & $\begin{array}{l}\text { Count } \\
(\mathrm{Lkc} / \mu \mathrm{L})\end{array}$ & $\begin{array}{c}\text { Recovery } \\
\text { (\%) }\end{array}$ \\
\hline 12.32 & -5.95 & 60.06 & -1.83 \\
\hline 53.68 & -14.46 & 95.70 & -15.20 \\
\hline 199.76 & -5.43 & 252.12 & 4.49 \\
\hline 228.80 & 10.17 & 900.00 & 1.17 \\
\hline
\end{tabular}

parameters, especially for specific gravity, proteins and urobilinogen while differences in urine sediment parameters were even more pronounced.

The disagreement of urine test strips analysis among different manufacturers has been already demonstrated, as well as discrepancy of urinalysis by automated urine analysers (12-14). Some previous studies based on comparison of various automated urine analysers have already highlighted major differences in their specifications and performances (14-17). Therefore, a certain level of disagreement of urine analysers developed by different manufacturers, based on diverse particle detection methods, was expected for this study.

Barbir et al. provided comparison of Atellica UAS 800 (Siemens healthineers, Erlangen, Germany) and Cobas u701 (Roche, Rotkreuz, Switzerland) analysers on a much smaller number of urine samples, in comparison to our study (18). They observed constant and proportional bias for erythrocyte and leukocyte counts, which is in accordance with results of our study. We have proved statistically significant constant bias for leukocytes and proportional bias for both erythrocytes and leukocytes. They also compared Atellica 1500 with microscopic urine sediment analysis using supravital staining which is an advantage over our study. Comparing to microscopic analysis they revealed proportional bias for erythrocytes and both constant and proportional bias for leukocytes counts. But our study encompassed a more detailed evaluation of Atellica 1500 specifications in addition to the comparison of Atellica 1500 with another urine 
analyser in their erythrocytes and leukocytes detection. We have successfully verified the following performance specifications of Atellica 1500 urine analyser: carryover, linearity, precision, reproducibility and LoB verification for erythrocyte and leukocyte counts. Additionally, the main advantage of our study is examined accuracy of Atellica UAS 800 and Iris urine analysers in their ability to detect bacteria.

Urinalysis is the first step in patient diagnostic pathway, where clinicians based on the following urine test strips and sediment results decide about further patient diagnostic examination and treatment. Along with the clinical symptoms of infections and presence of leukocytes in urine samples, bacteriuria is one of the main signs of urinary tract infections (UTIs) (3). Patients with present bacteriuria detected by routine urinalysis need to be sent for further urine culture. Foudraine et al. assessed great utility of iQ200 (Beckman Coulter, Brea, USA) parameters in combination with clinical symptoms and urine culture to predict or rule out UTIs (19). The number of urine culture analysis depends on bacteria results obtained by urine analysers. Therefore, it is of utmost importance to precisely and accurate detect bacteria. The accuracy of bacteria detection of Atellica UAS 800 and iQ200 analysers comparing with urine culture results showed intriguing results. Statistically significant difference in specificity of bacteria detection by these two urine analysers was observed. Iris analyser more specifically detects bacteria comparing to

\section{References}

1. Szwed JJ, Schaust C. The importance of microscopic examination of the urinary sediment. Am J Med Technol. 1982;48:141-3.

2. National Institution for Health and Care Excellence (NICE). Chronic kidney disease in adults: assessment and management; Clinical guideline. Available from: https://www.nice. org.uk/guidance/cg182. Accessed July 5th 2019.

3. Public Health England. Diagnosis of urinary tract infections (UTIs). Quick reference guide for primary care: For consultation and local adaptation. Available from: www.gov.uk/government/consultations/diagnosis-of-urinary-tract-infections-quick-reference-guide. Accessed July 5th 2019.
Atellica. But there was no statistically significant difference in their sensitivity of bacteria detection. Accurate bacteria detection is important to minimize the number of false positives and limit inappropriate antibiotic use.

During the performance of this study, we have noticed an inconsistency among urine analysers that may represent a potential source of aggravated interpretation of urine analysis results. The great discrepancy of reference ranges suggested by manufacturers was obtained. For example, negative bacteria results were $<6.8 \mathrm{p} / \mu \mathrm{L}$ for Iris, and $<$ $195.03 \mathrm{p} / \mu \mathrm{L}$ for Atellica. It is important that clinicians are familiar with this problem so that they can properly interpret results of urinalysis and provide adequate medical care of patients.

The absence of manual microscopic urine test results is a possible limitation of this study.

In conclusion, Atellica 1500 urine analyser meets manufacturer's quality criteria and has satisfactory analytical performance. There are large differences between Atellica 1500 and Iris analysers, due to which these two instruments are not comparable and can not be used interchangeably. Additionally, while there was no difference in specificity of bacteria detection between these two urine analysers, Iris analyser had greater sensitivity in bacteria detection.

\section{Potential conflict of interest}

None declared.
4. The American Urological Association. Renal Mass and Localized Renal Cancer: AUA Guideline. Available from: https:// www.auanet.org/guidelines/renal-cancer-renal-mass-andlocalized-renal-cancer-guideline\#x7068. Accessed July 5th 2019.

5. Bilic-Zulle L. Automatization of urine analysis. Biochem Med (Zagreb). 2018;28:S49-S50.

6. Kozic I, Zlabravec S, Perkovic Radojkovic K, Todoric Z, Sikirica $M$. Experience in automated urinalysis in private laboratory's daily routine. Biochem Med (Zagreb). 2018;28:S91-S92. 
7. McHugh ML. Interrater reliability: the kappa statistic. Biochem Med (Zagreb). 2012;22:276-82. https://doi. org/10.11613/BM.2012.031

8. Briggs C, Culp N, Davis B, D'Onofrio G, Zini G, Machin SJ, on behalf of the International Council for Standardization in Haematology. ICHS guidelines for the evaluation of blood cell analysers including those used for differential leucocyte and reticulocyte counting. Int J Lab Hematol. 2014;36:61327. https://doi.org/10.1111/ijlh.12201

9. Broughton PM, Gowenlock AH, McCormack JJ, Neill DW. A revised scheme for the evaluation of automatic instruments for use in clinical chemistry. Ann Clin Biochem. 1974;11:20718. https://doi.org/10.1177/000456327401100164

10. Clinical and Laboratory Standards Institute (CLSI). User Verification of Precision and Estimation of Bias, 3rd edition. CLSI document EP15-A3. Wayne,PA:CLSI,2014.

11. Clinical and Laboratory Standards Institute (CLSI). Evaluation of Detection Capability for Clinical Laboratory Measurement Procedures, 2nd edition. CLSI document EP17-A2. Wayne,PA:CLSI,2012.

12. Vuljanić $D$, Dojder A, Spoljaric V, Saracevic A, Dukic L, Lenicek Krleza J, et al. Analytical verification of 12 most commonly used urine dipsticks in Croatia: comparability, repetability and accuracy. Biochem Med (Zagreb). 2019;29:010708. https://doi.org/10.11613/BM.2019.010708

13. Vuljanic D, Dojder A, Spoljaric V, Saracevic A, Dukic L, Lenicek Krleza J, et al. Analytical verification of 12 most commonly used urine dipsticks in Croatia: comparability, reproducibility and accuracy. Biochem Med (Zagreb). 2018;28:S189-S190.
14. Oyaert M, Delanghe J. Progress in automated urinalysis. Ann Lab Med. 2019;39:15-22. https://doi.org/10.3343/ alm.2019.39.1.15

15. Ince $F D$, Ellidağ $H Y$, Koseoğlu $M$, Şimşek N, Yalçın H, Zengin $M O$. The comparison of automated urine analysers with manual microscopic examination for urinalysis automated urine analysers and manual urinalysis. Pract Lab Med. 2016;5:14-20. https://doi.org/10.1016/j.plabm.2016.03.002

16. Bakan E, Bayraktutan Z, Kilic Baygutalp N, Ali Gul M, Zuhal Umudum F, Bakan N. Evaluation of the analytical performances of Cobas 6500 and Sysmex UN series automated urinalysis systems with manual microscopic particle counting. Biochem Med (Zagreb). 2018;28:020712. https://doi. org/10.11613/BM.2018.020712

17. Laiwejpithaya S, Wongkrajang P, Reesukumal K, Bucha $C$ Meepanya S, Pattanavin C, et al. UriSed 3 and UX-2000 automated urine sediment analysers vs manual microscopic method: A comparative performance analysis. J Clin Lab Anal. 2018;32:e22249. https://doi.org/10.1002/jcla.22249

18. Barbir R, Perkov S, Taradi I. Significance of automation in standardization of qualitative urinalysis. Biochem Med (Zagreb). 2018;28:S156-S157.

19. Foudraine $D E$, Bauer MP, Russcher $A$, Kusters $E$, Cobbaert $C M$, van der Beek MT, Stalenhoef JE. Use of automated urine microscopy analysis in clinical diagnosis of urinary tract infection: defining an optimal diagnostic score in an academic medical center population. J Clin Microbiol. 2018;56:e02030-17. https://doi.org/10.1128/JCM.02030-17 